Article

\title{
Indirect Taxis on a Fluctuating Environment
}

\author{
Andrey Morgulis ${ }^{1,2, *,+}$ and Konstantin Ilin ${ }^{3,+}$ \\ 1 I.I.Vorovich Institute for Mathematic, Mechanics and Computer Science, Southern Federal University, \\ Rostov-na-Donu 344090, Russia \\ 2 Southern Mathematical Institute of VSC RAS, Vladikavkaz 362027, Russia \\ 3 Department of Math, The University of York, Heslington YO10 5DD, UK; konstantin.ilin@york.ac.uk \\ * Correspondence: morgulisandrey@gmail.com \\ + Both authors contributed equally to this work.
}

Received: 28 September 2020; Accepted: 12 November 2020; Published: 17 November 2020

check for updates

\begin{abstract}
In this article, we study a Patlak-Keller-Siegel (PKS) model of a community of two species placed in the inhomogeneous environment. We employ PKS law for modeling tactic movement due to interspecific taxis and in response to the environmental fluctuations. These fluctuations can arise for natural reasons, e.g., the terrain relief, the sea currents and the food resource distribution, and there are artificial ones. The main result in the article elucidates the effect of the small-scale environmental fluctuations on the large-scale pattern formation in PKS systems. This issue remains uncharted, although numerous studies have addressed the pattern formation while assuming an homogeneous environment. Meanwhile, exploring the role of the fluctuating environment is substantial in many respects, for instance, for predicting the side effects of human activity or for designing the control of biological systems. As well, it is necessary for understanding the roles played in the dynamics of trophic communities by the natural environmental inhomogeneities-those mentioned above, for example. We examined the small-scale environmental inhomogeneities in the spirit of Kapitza's theory of the upside-down pendulum, but we used the homogenization instead of classical averaging. This approach is novel for the dynamics of PKS systems (though used commonly for other areas). Employing it has unveiled a novel mechanism of exerting the effect from the fluctuating environment on the pattern formation by the drift of species arising upon the homogenization of the fluctuations.
\end{abstract}

Keywords: Patlak-Keller-Segel systems; stability; instability; bifurcation; averaging; homogenization

\section{Introduction}

Mathematical modeling of the pattern formation by the active media widely has employed the Patlak-Keller-Segel (PKS) systems for several decades. The reviews [1-4] give insights into the progress made by the theory and applications of such systems during the last half-century. The formulations of PKS system typically possess the translation invariance, and the translation-invariant equilibrium, therefore. This state is homogeneous in the sense that the density of every species is constant. Bringing the system out of such an equilibrium is necessary for the non-trivial spatiotemporal patterns. An evident reason for getting out the equilibrium is the instability of it to small perturbations. The local bifurcations accompanying the occurrence of such instabilities can give rise to the non-trivial patterns. For instance, Berezovskaya and Karev [5,6] reported this way for the traveling waves in PKS systems to occur. At the same time, it is still unclear whether there are any links from the local bifurcations to the semi-soliton waves reported in articles [7-9] or the waves with iterated-exponential convergence to the limit values at the infinity [10]. Nevertheless, from the observations reported in articles [11-15], it follows that the instabilities of equilibria accompanied by the local bifurcations often are the first links in the chains of dynamical transitions leading to rather complex spatiotemporal patterns. 
It is natural to see the translational invariance of a PKS system as a formalization of either assuming the homogeneity of the environment or neglecting the response of the species to the existing inhomogeneity. However, in the biological literature, there are many pieces of evidence of how substantial such responses can be. For instance, Southall et al. [16] reported the disorienting effect of anthropogenic acoustic noises on marine mammals. As well, several articles report the effects of thermal fronts and mesoscale oceanic eddies on the distributions of fish populations [17-19]. One more effect is due to switching omnivores to an alternative feeding resource [20]. It is natural to suppose inhomogeneity regarding a mathematical model aimed at studying such kinds of effects. Additionally, considering the inhomogeneity can be crucial for addressing the issues of the control design for the biological systems or predicting the side effects of various human activities.

For an inhomogeneous PKS system, it is natural to raise a question concerned with the pattern formation in a way similar to that we have been mentioning for the case of homogeneity. Here we mean the transitions caused by instabilities of some counterparts of the homogeneous equilibrium. Given this analogy, it is also natural to ask to what extent the inhomogeneities influence these instabilities compared to the case of homogeneity. These questions become quite tractable provided that the scale of the environmental fluctuations is relatively small as we can address them by borrowing the ideas of Kapitza's theory of the upside-down pendulum [21] and combining them with the homogenization [22,23]. Nevertheless, this area is almost unexplored. In general, the inhomogeneous PKS models get much less attention than the homogeneous ones, and besides, those few articles which treat the case of inhomogeneity consider the global boundedness, the extinction or coexistence, e.g., [24,25], but not the issues we have been raising above. The only exception we are aware of is the article [26] that employs the homogenization for studying the effect of the terrain relief on the excitation of waves in a spatially distributed living community. However, it does not account for the tactic transport.

In the present article, we explore a community of two species placed in the inhomogeneous environment. We assume that one of these species responds by tactic movements to the inhomogeneity of distribution of the other species and an external signal that mimics the environmental fluctuations. At that, both kinds of response obey the PKS law. For the short-wavelength external signal, we study the effect of it on the large-scale patterns following the plan outlined above. It turns out that the only conductor of this effect is a drift of species. When the external signal takes the form of a traveling wave, there exist the quasi-equilibria, which are "equilibria on average", roughly speaking. Examining the stability of the quasi-equilibria shows that, compared to the case of the homogeneous environment, increasing the magnitude of the fluctuations can destabilize the quasi-equilibria and stabilize them. Which of these effects occurs depends only on the speed at which the signal-making wave propagates, but they both are exponential in the amplitude of the wave.

The article consists of six sections supplemented with three appendices. In Section 2, we formulate the governing equations. In Section 3, we address the short-wavelength external signals and describe the homogenized system. In Section 4 , we introduce the equilibria and quasi-equilibria and explore the stability and instability of them. In Section 5, we discuss stabilizing and destabilizing the quasi-equilibria due to the external signal. Section 6 contains several final remarks. Appendixes A-C contain the details of proofs of the assertions formulated in the article body.

\section{The Governing Equations}

We will be considering a model of the indirect taxis that reads as

$$
\begin{aligned}
& \phi_{t}=q+\kappa^{-1} f-v \phi+\delta_{1} \phi_{x x} ; \\
& p_{t}=\left(\delta_{2} p_{x}-\kappa p \phi_{x}\right)_{x} ; \\
& q_{t}=q(1-q-p)+\delta q_{x x} .
\end{aligned}
$$


Here, $x, t$ stand for the coordinates in space and time, correspondingly, or while being used as subscripts, the partial differentiation in the corresponding coordinate. $\delta_{1}, \delta_{2}, \delta, \kappa$ and $v$ stand for the positive parameters.

Equations (2) and (3) describe the balances of densities of two interacting species. At that, the former involves the PKS cross-diffusion while the latter does not. In what follows, the species possessing (not possessing) taxis stands as the predator (prey). We denote the densities of the former and the latter as $p$ and $q$, correspondingly. The conservatism in the predators' balance relies on assuming the negligibly slow reproduction and mortality of them compared to the other processes considered. The kinetics of prey follow the logistic and Lotka-Volterra laws.

Note that the stimulus driving the tactic movements of the predators is not the prey density itself but another signal, the intensity of which we have been denoting as $\phi$. The notation $\kappa$ stands for the coefficient that measures the tactic efficiency. In this sense, the prey-taxis is indirect. Equation (1) governs the production of the signal, which gets an external contribution described by the given function, $f=f(x, t)$.

Tello and Wrozhek and also Li and Tao, have addressed the indirect taxis in their recent articles [27,28]. They were focusing upon the existence of the non-trivial steady states and the global boundedness of solutions. Tyutyunov et al. [29], also recently, have noticed the equivalence between the system (1)-(3) and that proposed earlier by Govorukhin et al. and by Arditi et al. in the articles [11,12] for taking into account the inertia of the tactic motions. The latter reads as

$$
\begin{aligned}
& u_{t}=(\kappa q+f)_{x}-v u+\delta_{1} u_{x x} \\
& p_{t}=\left(\delta_{2} p_{x}-p u\right)_{x} \\
& q_{t}=q(1-q-p)+\delta q_{x x} .
\end{aligned}
$$

Bringing system (1)-(3) at the inertial form (4)-(6) employs a simple ansatz $u=\kappa \phi_{x}$. It introduces new dependent variable denoted as $u$, which is nothing other than the velocity of the predator's advection. Equation (4) governs this velocity in response to the prey density and the external signal. Additionally, it takes into account the velocity diffusion and the resistance to the predator's motion due to the environment, and the coefficients $\delta_{1}$ and $v$ measure the intensities of the former and latter correspondingly. In some respects, the inertial system (4)-(6) is similar to the Cattaneo model of chemosensitive motions and the hyperbolic limits for the kinetic equations discussed in the articles [30,31].

We pay attention to the formulated equivalence, since in the above-cited articles, Govorukhin et al. and Arditi et al. reported the transitions to the complex wave motions due to the destabilization of the homogeneous equilibria of the homogeneous version of the inertial system (4)-(6) (in which $f=0$ ). We will be considering only the inertial system (4)-(6) henceforth.

\section{Homogenization and Drift}

In what follows, let us consider fast variables $(\xi, \tau)$ as the angular coordinates on 2-torus $\mathbb{T}^{2}$. Define

$$
\langle g\rangle(x, t)=\frac{1}{4 \pi} \int_{0}^{2 \pi} \int_{0}^{2 \pi} g(x, t, \xi, \tau) d \xi d \tau .
$$

Theorem 1. Let the external signal in Equation (4) take a short-wavelength form, i.e.,

$$
f=f(x, t, \xi, \tau), \xi=\omega x, \tau=\omega t, \omega \rightarrow \infty,
$$

and let the diffusion rates in Equations (4) and (5) be of the same order as the wave length, namely:

$$
\delta_{1}=v_{1} \omega^{-1}, \delta_{2}=v_{2} \omega^{-1}, v_{1}=\text { const }>0, v_{2}=\text { const }>0 .
$$


We state that under assumptions (8) and (9) the shortwave asymptote of system (4)-(6) takes the following form

$$
\begin{gathered}
q(x, t)=\bar{q}(x, t)+O\left(\omega^{-1}\right), \omega \rightarrow+\infty ; \\
u(x, t)=\bar{u}(x, t)+\widetilde{u}(x, t, \tau, \tilde{\xi})+O\left(\omega^{-1}\right), \omega \rightarrow+\infty ; \\
p(x, t)=\bar{p}(x, t) P(x, t, \tau, \xi)+O\left(\omega^{-1}\right), \omega \rightarrow+\infty ; \\
\widetilde{u}_{\tau}=\left(f+v_{1} \widetilde{u}_{\xi}\right)_{\xi},\langle\widetilde{u}\rangle=0 ; \\
P_{\tau}=\left(v_{2} P_{\xi}-P(\bar{u}+\widetilde{u})\right)_{\tilde{\xi}},\langle P\rangle=1 ; \\
\bar{u}_{t}=(\kappa \bar{q}+\bar{f})_{x}-v \bar{u}, \bar{f}=\langle f\rangle ; \\
\bar{p}_{t}+(\bar{p}(\bar{u}+\langle\widetilde{u} P\rangle))_{x}=0 ; \\
\bar{q}_{t}=\bar{q}(1-\bar{p}-\bar{q})+\delta \bar{q}_{x x}
\end{gathered}
$$

where the problems (13) and (14) have to be solved on $\mathbb{T}^{2}$.

We place the proof into Appendix A.

In what follows, we will be calling the system of Equations (15)-(17) homogenized. The homogenized system is closed relative to unknowns $\bar{p}, \bar{q}$ and $\bar{u}$ for every specific external signal. Indeed, once we have specified the external signal, $f$, Equation (13) determines the shortwave velocity denoted as $\widetilde{u}$, which in turn enters Equation (14) as the coefficient. Then by resolving the problem (14) and calculating the value of $\langle\widetilde{u} P\rangle$, we determine a mapping $\bar{u} \mapsto\langle\widetilde{u} P\rangle$ that acts between the functions in the slow variables, $x, t$. Hence, Equation (16) links the unknowns $\bar{u}$ and $\bar{p}$ in a non-local manner.

From Equation (16), it follows that the averaged velocity, $\bar{u}$, is not the actual velocity of predators' advection-that is, there is some drift, the velocity of which is equal to $\langle\widetilde{u} P\rangle$. This drift collects all the remembrances about the external signal and conducts them to the homogenized system.

We will be using the following notation and auxiliary notions. Let $f$ be given; we call the mapping $\mathcal{V}(f): \bar{u} \mapsto\langle\widetilde{u} P\rangle$ the drift operator. Let $\mathcal{G}: f \mapsto \widetilde{u}$ be the transformation resulting from resolving problem (13). Further, let $\sigma=$ const $\in \mathbb{R}$ and smooth function $\widetilde{u}=\widetilde{u}(x, t, \xi, \tau)$ be given, and moreover, let $\langle\widetilde{u}\rangle=0$. We define the mapping

$$
\mathcal{P}(\widetilde{u}): \sigma \mapsto\langle\widetilde{u} P\rangle,
$$

where $P=P(x, t, \xi, \tau)$ is the solution to problem

$$
P_{\tau}=\left(v_{2} \partial_{\xi} P-P(\sigma+\widetilde{u})\right)_{\xi}, \quad\langle P\rangle=1
$$

Problem (19) has a unique solution for every $\sigma, \widetilde{u}$ (by a lemma proved in Appendix A). Once the function $\widetilde{u}$ is prescribed, the mapping (18) parameterizes a path in a suitable space of functions in variables $(x, t)$. Finally, we arrive at the identity

$$
(\mathcal{V}(f) \bar{u})(x, t)=\mathcal{P}(\mathcal{G} f) \bar{u}(x, t)
$$

For every signal $f$ there exists a decomposition $f=\bar{f}+\widetilde{f}$, where $\bar{f}=\langle f\rangle$. From the setting of the problem (13), it follows that $\mathcal{V}(f)=\mathcal{V}(\widetilde{f})$. Hereafter we will be using the notation $\mathcal{V}(\widetilde{f})$ for the drift operator.

Example 1. Let us consider an external signal that has the form of the short traveling wave-that is, let us put

$$
f=f(x, t, \xi, \tau)=\bar{f}(x, t)+A g(x, t, \eta), \quad \eta=\xi-c \tau
$$

where $A=$ const $\geq 0, c=$ const $\geq 0$ and the signal profile, $g(x, t, \cdot)$, is a smooth $2 \pi$-periodic function that is equal to zero on average for every $(x, t)$ (while considering the short-wavelength limit for these waves, we require 
$2 \pi /$ c-periodicity in $\tau$ instead of $2 \pi$-periodicity; in accordance with this, we re-define the averaging, $\langle\cdot\rangle)$. Hence, we have $\widetilde{f}=A g$.Let the notation of $\partial_{\eta}^{-1}$ stand for the right inverse to the differentiation $\partial_{\eta}$ - that is,

$$
\partial_{\eta} \partial_{\eta}^{-1} w=w, \int_{0}^{2 \pi} \partial_{\eta}^{-1} w d \eta=0 \quad \forall w: w(\cdot, \eta, \cdot)=w(\cdot, \eta+2 \pi, \cdot), \int_{0}^{2 \pi} w d \eta=0 .
$$

Let $q * h$ denote the common convolution of functions $q$ and $h$ on the real axis. Define

$$
\begin{gathered}
\exp _{ \pm}(\sigma)=\left\{\begin{array}{c}
\mathrm{e}^{\sigma}, \pm \sigma>0, \\
0, \mp \sigma<0,
\end{array}\right. \\
s(x, t, \cdot)=-\partial_{\eta}^{-1} \exp _{+}^{-\frac{c}{v_{1}}} * g(x, t, \cdot) ;
\end{gathered}
$$

where the superscript indicates raising to a power, and the convolution acts in variable $\eta$. Then

$$
\widetilde{u}=A \mathcal{G} g=v_{1}^{-1} A \partial_{\eta} s
$$

Further, we put

$$
\begin{gathered}
E(x, t, \cdot)=\mathrm{e}^{a s}, a=\left(v_{1} v_{2}\right)^{-1} A, \\
R(x, t, \sigma)=\left\langle\frac{E(x, t, \cdot)}{E(x, t,-\sigma)}\right\rangle, \\
z=\frac{c-\bar{u}(x, t)}{v_{2}} .
\end{gathered}
$$

Let $z \neq 0$. The periodic solution to Equation (14) has the form

$$
\begin{gathered}
P(x, t, z, \cdot)=\Gamma_{ \pm}^{-1}(x, t, z) E(x, t, \cdot)\left(\exp _{ \pm}^{-z} * E^{-1}(x, t, \cdot)\right), \quad \pm z>0 \\
\Gamma_{ \pm}(x, t, z)=\int_{\mathbb{R}} \exp _{ \pm}^{-z}(\sigma) R(x, t, \sigma) d \sigma, \pm z>0 .
\end{gathered}
$$

Using the Fourier series matches two expressions shown in (29) one to another one. Indeed, let $\hat{E}_{k}(x, t)$ and $\check{E}_{k}(x, t)$ be the Fourier coefficients of functions $E(x, t, \cdot)$ and $E^{-1}(x, t, \cdot)$ correspondingly. Then

$$
\begin{gathered}
P(x, t, z, \eta)=\Gamma^{-1}(x, t, z) E(x, t, \eta)\left(\check{E}_{0}(x, t)+z \sum_{k \in \mathbb{Z} \backslash\{0\}} \frac{\check{E}_{k}(x, t) e^{i k \eta}}{z+i k}\right), \\
\Gamma(x, t, z)=\left(\check{E}_{0} \hat{E}_{0}\right)(x, t)+z \sum_{k \in \mathbb{Z} \backslash\{0\}} \frac{\left(\check{E}_{k}^{*} \breve{k}_{k}\right)(x, t)}{z+i k} .
\end{gathered}
$$

From the expressions (24), (25) and (31), it follows that

$$
\mathcal{V}(\widetilde{f}) \bar{u}=\langle\widetilde{u} P\rangle=-v_{2} z \Gamma^{-1}(x, t, z) \sum_{k \in \mathbb{Z} \backslash\{0\}} \frac{i k\left(\hat{E}_{k}^{*} \check{E}_{k}\right)}{z+i k}=v_{2} z\left(1-\Gamma^{-1}(x, t, z)\right) .
$$

For deriving the second equality in this chain, we transform the central expression of it with the use of the equalities $i k(z+i k)^{-1}=1-z(z+i k)^{-1}$,

$$
\check{E}_{0} \hat{E}_{0}+\sum_{k \neq 0} \hat{E}_{k}^{*} \check{E}_{k}=\left\langle E E^{-1}\right\rangle=1
$$

and the Formula (32). Further, the total velocity of predators' transport that enters Equation (16) reads as

$$
v \stackrel{\text { def }}{=} \bar{u}+\mathcal{V}(\widetilde{f}) \bar{u}=\bar{u}+\langle\widetilde{u} P\rangle .
$$


By Equality (33),

$$
v=v(x, t, z)=c-\frac{z v_{2}}{\Gamma(x, t, z)} .
$$

Since

$$
\Gamma(x, t, z)= \pm z \Gamma_{ \pm}(x, t, z), \pm z>0
$$

we get additional representations for the total transport velocity, namely

$$
v(x, t, z)=c \mp \frac{v_{2}}{\Gamma_{ \pm}(x, t, z)}, \pm z>0 .
$$

Expressions (32) and (35) give the analytic continuation to the advective velocity to some strip parallel to the real axis in the complex plane of variable $z$. This feature is a particular manifestation of the analyticity of the drift operator. We shall put it more accurately in Appendix B while proving Lemma 1.

Remark 1. Formula (36) (where one has to put $z=c / v_{2}>0$ ) shows that zeroing the averaged predator' velocity, $\bar{u}$, does not entail zeroing the total transport velocity, $v$. Indeed,

$$
v\left(x, t, c / v_{2}\right)=\mathcal{V}(\widetilde{f}) 0=c-\frac{v_{2}}{\Gamma_{+}\left(x, t, c / v_{2}\right)} \stackrel{\text { def }}{=} v_{e}(x, t), c>0 .
$$

Thus, the advection of the predators generically goes on even if the averaged velocity, $\bar{u}$, vanishes, at least provided that the external signal belongs to the class of the traveling waves introduced by the Formula (21). There exists residual drifting; the velocity of that takes the form determined by the Equality (37). The only exception is the case of $c=0$. Inspecting it with the use of Formulae (33) and (35) shows that $v=\mathcal{V}(\widetilde{f}) 0=0$. Hence, a stationary signal produces no residual drift.

\section{Stability Analysis}

At this point, several terminological remarks and comments seem to be useful. Basically, our understanding of the notion of stability comes from Liapunov's theory. In particular, the linear stability analysis for an equilibrium of real-valued system $\dot{y}=F(y)$ means studying the behavior of the small perturbations having the form $\exp (\lambda t) y$, or, for more generality, the semi-group $\exp (t A)$ generated by the differential $A$ of the mapping $F$ evaluated at this equilibrium. In particular, the solutions of the spectral problem $A y=\lambda y$ are in one-to-one correspondence to the eigenmodes of the small perturbations having the above form. We call such a spectral problem the spectral stability problem. We say that an eigenmode is stable (unstable, neutral) if the real part of the corresponding eigenvalue, $\lambda$, is negative (positive, equal to zero).

Let some system, together with the equilibrium of it, smoothly depend on several parameters. The space of the parameters naturally separates itself into the areas of instability or stability. When a point of parameters' space belongs to the former (latter) area, there exist (no) unstable eigenmodes. Every smooth path embedded in the space of the parameters parameterizes the family of equilibria, which in turn generates the family of the stability problems. We call a submanifold in the space of the parameters neutral if the transversal intersection of it by a smooth path generically entails the transversal intersection of the imaginary axis by the branch of the eigenvalues of the corresponding stability problems. In general, the neutral submanifold of a specific equilibrium is multi-foliated or stratified. Disappearing or arising unstable eigenmodes, doing so upon crossing the neutral submanifold, are what we call occurrences of instability. We say that the instability is oscillatory (monotonic) if the corresponding branch of the eigenvalues is not real (is real). We call critical the subset of neutral submanifold that separates the areas of stability and instability one from another one.

It is well-known that an occurrence of instability in the family of equilibria of a smooth family of vector fields indicates the local bifurcations. If there are no additional degenerations then branching the equilibria family accompanies the monotone instability, and branching the limit cycle off the 
family accompanies the oscillatory instability. The latter is the so-called Poincare-Andronov-Hopf bifurcation. More complex bifurcations happen in the case of additional degeneracy, e.g., when the neutral spectrum is multiple. The monographs [32-34] provides a great piece of information on this subject.

\subsection{Equilibria and Quasi-Equilibria}

By equilibrium, we mean the solution to the exact system (4)-(6) that reads as

$$
p \equiv p_{e}, \quad q \equiv q_{e}, u=0, \quad p_{e}=\text { const }>0, \quad q_{e}=\text { const }>0, \quad p_{e}+q_{e}=1 .
$$

When the exact system possesses translational invariance, it can stay in every equilibrium of family (38). If the homogenized system (15)-(17) has a solution of the family (38), we call this solution quasi-equilibrium. For a quasi-equilibrium (if any), Formulae (10)-(14) determine the leading approximation to a short-wavelength pattern that represents an equilibrium "on average". In the considerations below, the quasi-equilibria and the related short-wavelength patterns are not distinguishable. Therefore we will be using the same name for both.

The following examples deliver the classes of signals which lead to the homogenized systems allowing quasi-equilibria.

Example 2. Let $f=\bar{f}+A g(x, t, \xi)$ where $A=\mathrm{const} \geq 0$, so that $\tilde{f}=A g$. Such a signal is a limit case of those defined by Formula (21) for $c=0$ and it is stationary in the fast variables. The corresponding short-wave pattern reads as

$$
\widetilde{u}=\widetilde{u}_{e}=-A\left(v_{1} \partial_{\tilde{\xi}}\right)^{-1} g, \quad P=P_{e}=\left\langle\mathrm{e}^{a s}\right\rangle^{-1} \mathrm{e}^{a s}, a=\left(v_{1} v_{2}\right)^{-1} A, s=-\partial_{\tilde{\xi}}^{-2} g .
$$

These expressions follow from Formulae (24), (25), (31) and (32) upon tending c to zero, or directly from solving the problems (13) and (14). As we have been saying in Remark 1, a stationary signal does not produce the residual drift entails $v_{e}=\mathcal{V}(\widetilde{f}) 0=0$. Hence, there exist the quasi-equilbria (38).

Example 3. Let $f=\widetilde{f}(t, \xi, \tau)$ where $\langle\widetilde{f}(t, \cdot)\rangle=0$ for every $t$. Then it is easy to see that the velocity of the residual drift, $v_{e}$, is invariant with respect to translations not in time but in space only. Nevertheless, it suffices for existing the quasi-equilibria (38). In particular, a signal having the form $\widetilde{f}=A g(t, \eta)$, where $\eta=\xi-c \tau$, $A=\mathrm{const} \geq 0, c=\mathrm{const} \geq 0$, belongs to the class of signals that has appeared as the Example 1 . Then the Formulae (24), (25), (29) and (37) upon making $z=c / v$ give us the explicit expressions for the quasi-equilibrium pattern and for the residual drift velocity. The corresponding quasi-equilibrium pattern is the short traveling wave that propagates at the same speed as the signal-making wave.

Consider an inhomogeneous system (4)-(6) where the external signal reads as

$$
f=\widetilde{f}=A g, g=g(\eta), \eta=\xi-c \tau, A=\text { const } \geq 0, c=\text { const } \geq 0 .
$$

The signals determined by Formula (39) belong to the intersection of the classes which we have been examining as Examples 1 and 3 . As we have been noticing, there exists the family of homogeneous quasi-equilibria (38) for every such a signal. Let the signal profile, $g$, be a smooth periodic function vanishing on average. If the signal profile remains invariable then there are only two parameters which control the effect of the signal. These are the wave speed, $c$, and the effective amplitude of it, $a$. We have defined the latter in the line (26). When the signal is off - that is, for $a=0$, the homogenized system becomes homogeneous with $\delta_{1}=\delta_{2}=0$. Correspondingly, the quasi-equilibria turn into the equilibria. We target studying the effect from gradual amplifying the signal on the stability and instability of quasi-equilibria compared to the equilibria. For this purpose, we will be watching the deformations of the neutral submanifold in the parametric space depending on the values of $a$ and $c$. 
We start with revisiting the onset of instability of the homogeneous equilibria, which is known mainly due to the works by Govorukhin et al. [11] and by Arditi et al. [12].

\subsection{Stability of Equilibria}

Let us choose out of family (38) an equilibrium with some specific densities $p_{e}>0$ and $q_{e}=1-p_{e}>0$. The system governing the evolution of a small perturbation of such an equilibrium reads as

$$
\begin{gathered}
u_{t}+v u-\kappa q_{x}=\delta_{1} u_{x x} \\
p_{t}+p_{e} u_{x}=\delta_{2} p_{x x} \\
q_{t}+q_{e}(p+q)=\delta q_{x x} \\
p_{e}+q_{e}=1 .
\end{gathered}
$$

Since the homogeneous system possesses the translational invariance, we will be considering only the so-called normal modes of small perturbations, which have following form.

$$
(\hat{u}, \hat{p}, \hat{q}) \exp (i \alpha x+\lambda t), \lambda=\lambda(\alpha) \in \mathbb{C}, \alpha \in \mathbb{R},
$$

where $\lambda$ is the eigenvalue of the corresponding spectral stability problem.

It is convenient to introduce the following notation

$$
\beta=\alpha^{2}, \bar{\delta}=\left(\nu, \delta, \delta_{1}, \delta_{2}\right) .
$$

Note that each equilibrium (38) has a neutral homogeneous mode (that corresponds to $\lambda=\alpha=0$ ), but this does not lead to any long-wave instabilities. To get rid of this and other unwanted degenerations, we assume the following

$$
\beta>0,0<p_{e}<1, v\left(\delta_{1}+\delta_{2}+\delta\right)>0 .
$$

Let $\Pi$ be a domain cut out by inequalities (44) in the space of parameters $p_{e}, \beta, \bar{\delta}$. Let us consider an equilibrium with a specific density $p_{e}$ and adjoin to it the eigenmodes (43). Then the following assertions are true.

(i) The neutral submanifold in the space of parameters represents the graph of function

$$
\kappa_{c}=\frac{\left(D_{1}+D_{2}\right)\left(D_{1}+D_{3}\right)\left(D_{2}+D_{3}\right)}{\beta p_{e} q_{e}} \text { where } D_{1}=v+\beta \delta_{1}, D_{2}=\beta \delta_{2}, D_{3}=q_{e}+\beta \delta .
$$

Every eigenmode having the wavenumbers $\alpha= \pm \sqrt{\beta}$ is stable provided that $\kappa<\kappa_{\mathcal{c}}\left(p_{e}, \beta, \bar{\delta}\right)$, and there is an unstable mode with the same wavenumbers provided that $\kappa>\kappa_{c}\left(p_{e}, \beta, \bar{\delta}\right)$.

(ii) The following inequality holds:

$$
\min _{0<p_{e}<1, \beta>0} \kappa_{\mathcal{c}}\left(p_{e}, \beta, \bar{\delta}\right)=\kappa_{*}(\bar{\delta})>0
$$

where the strict positiveness takes place for every $\bar{\delta}$ obeying the restrictions (44). If $\kappa<\kappa_{*}(\bar{\delta})$ then the collective stability takes place in the sense that every eigenmode linked to every equilibria is stable; i.e., the eigenmodes are stable for every admissible values of $p_{e}$ and $\alpha$. 
The first subsection of Appendix $C$ contains the details of the stability analysis. Checking the positiveness of the quantity $\kappa_{*}(\bar{\delta})$ is just direct inspecting of the explicit expression (45) including the case of $\delta_{1}=\delta_{2}=0$ closely relevant to the quasi-equilibria. We put

$$
\begin{gathered}
\kappa_{c}^{(0)}\left(p_{e}, \beta, v, \delta\right)=\left.\kappa_{c}\left(p_{e}, \beta, \bar{\delta}\right)\right|_{\delta_{1}=\delta_{2}=0}=\frac{v\left(v+q_{e}+\beta \delta\right)\left(q_{e}+\beta \delta\right)}{\beta p_{e} q_{e}}, \\
\kappa_{*}^{(0)}(v, \delta)=\min _{0<p_{e}<1, \beta>0} \kappa_{c}^{(0)}=\left.\kappa_{*}(\bar{\delta})\right|_{\delta_{1}=\delta_{2}=0}>0 .
\end{gathered}
$$

For instance, $\kappa_{*}^{(0)}(1,1)=27 / 2$. At that, the coordinates of minimizer are $p_{e}=\beta=2 / 3$.

In Figure 1, the box (a) depicts the areas of stability and instability in the space of parameters $p_{e}, \beta, \kappa$ for $\bar{\delta}=(1,1,0,0)$ by visualizing the neutral surface that separates them. This is the graph of the quantity $\left.\kappa_{c}^{(0)}\right|_{\nu=\delta=1}$ vs $p_{e}, \beta$. An individual eigenmode linked to a specific equilibrium gets unstable (stable) when the corresponding triple $p_{e}, \beta, \kappa$ gets above (beneath) this graph. Below the lowest point of it there is a layer, the whole of which is inside the stability area. This inclusion matches clause (ii) above that asserts the collective stability. This time the collective stability takes place provided that $\kappa<\kappa_{*}(1,1,0,0)=\kappa_{*}^{(0)}(1,1)=27 / 2$. For every $\kappa>\kappa_{*}$, every level set $\kappa=\kappa_{\mathcal{c}}$ determines a closed neutral curve inside the semi-strip $\left\{0<p_{e}<1, \beta>0\right\}$. Thus increasing the value of $p_{e}$ inevitably destabilizes the eigenmodes in some range of wavenumbers provided that $\kappa>\kappa_{*}$. When $\kappa \rightarrow+\infty$, the corresponding neutral curve widens itself and tends to the boundary of the semi-strip. The shape of the pictured neutral surface is typical for the non-zero $\delta_{1}$ and $\delta_{2}$ as well.

The neutral spectrum arising on the neutral submanifold is multiple. Namely, the oscillatory eigenmode is double due to the mirror symmetry. Additionally, there is a simple null eigenvalue that exists for every admissible set of the problem parameters as well. This degeneration arises from the conservation law for predators' density, because of which the homogeneous equilibria are not isolated but form the continuous 1-parametric family.

In the mentioned articles, Govorukhin et al. and Arditi et al. studied the occurrence of instability for the homogeneous system in a confined environment with Neumann's boundary conditions (also known as the no-flux boundary conditions). Imposing these boundary conditions removes the mirror symmetry and the multiplicity of the oscillatory modes, but the conservation law, the equilibria family and the null eigenvalue persist. This residual degeneration does not cause serious difficulty. One can get rid of it by restricting the system on the level sets of the conserved quantity. As a result, the common theory of the Poincare-Andronov-Hopf bifurcation becomes applicable to this restriction. Alternatively, one can apply the general results on the bifurcation accompanying the oscillatory instability in the family of vector fields, which possess the so-called cosymmetry [35]. (Given the cosymmetry, the branching of limit cycle generically sharply differs from the Poincare-Andronov-Hopf scenario except for the case of so-called integrable cosymmetry that is equivalent to a conservation law. In case of such an exception, the cycle branches off, roughly speaking, "as usual" provided that there is no additional degeneration.) Furthermore, the direct numerical simulations performed by the same authors demonstrate exciting the waves due to the above instabilities and bifurcations. At that, predators' density, $p_{e}$, plays the part of the control parameter. These waves are time-periodical for the weakly supercritical values of $p_{e}$ and tend to move chaotically upon increasing the value of $p_{e}$. Additionally, it turns out that the wavy motions allow the predators to consume more while keeping the stock of prey greater. In this sense, the waves are always more advantageous than equilibria. At this point, we arrive at seeing the collective stability as a failure of the adaptation to lacking the resource due to insufficient tactic intensity.

The instability of equilibria of system (4)-(6) arises from only the indirectness (or inertia) of taxis. It is never monotonous. In contrast, direct taxis rather leads to monotone instability. For example, the instability is necessarily monotonous for the homogeneous equilibria of so-called classical PKS systems listed down by Hillen and Painter [3]. This kind of instability is also able to launch transiting to the complex patterns $[13,36]$. Generally, of course, a competition between both type of instabilities 
takes place. In particular, the articles $[5,6,37]$ reported several scenarios of the traveling waves in the auto-tactic systems due to both types of instabilities. At that, the oscillatory instability turned out to require more complex kinetics and tactic interactions than the monotone one. Several authors $[14,15]$ have explored the instabilities and pattern formation in more general PKS models which involve sophisticated kinetic and tactic interplays of more than two species in the homogeneous environment confined within the no-flux boundaries.

\subsection{Stability of the Quasi-Equilibria}

Lemma 1. Let a smooth external signal produce a quasi-equilibrium with densities $p_{e}>0$ and $q_{e}=1-p_{e}>0$. The linear problem governing the small perturbations of this quasi-equilibrium reads as

$$
\begin{gathered}
\bar{u}_{t}+v \bar{u}-\kappa \bar{q}_{x}=0 ; \\
\bar{p}_{t}+\left(v_{e} \bar{p}+p_{e}\left(1+\mathcal{V}^{\prime}(\widetilde{f})\right) \bar{u}\right)_{x}=0 ; \\
\bar{q}_{t}+q_{e}(\bar{p}+\bar{q})-\delta \bar{q}_{x x}=0
\end{gathered}
$$

where the residual drift velocity, $v_{e}=\mathcal{V}(\widetilde{f}) 0$, generally is a real valued function in variables $(x, t)$. The notation of $\mathcal{V}^{\prime}(\widetilde{f})$ stands for the differential of the drift operator evaluated at $\bar{u}=0$. This differential acts by multiplying by a real-valued function in variables $(x, t)$. Namely,

$$
\begin{gathered}
v_{e}=\mathcal{V}(\widetilde{f}) 0=\left\langle\widetilde{u} P_{e}\right\rangle, \quad \mathcal{V}^{\prime}(\widetilde{f}): g \mapsto\left\langle\widetilde{u} P_{1}\right\rangle g, \text { where } \\
P_{1 \tau}=\left(v_{2} P_{1 \xi}-P_{e}-\widetilde{u} P_{1}\right)_{\xi},\left\langle P_{1}\right\rangle=0, \quad P_{e \tau}=\left(v_{2} P_{e \xi}-\widetilde{u} P_{e}\right)_{\xi},\left\langle P_{e}\right\rangle=1
\end{gathered}
$$

where $\widetilde{u}$ arises from solving the problem (13).

Proof. Deriving the expressions claimed by the lemma is straightforward except for the differentiability of the drift operator in the origin. In fact, it is analytic there, and this is what allows us to get Formulae (52) and (53) by the common perturbation technique. We postpone proving the analyticity up to Appendix B.

Lemma 2. Let the external signal, $f$, take the form determined by expression (39). Then there exists the quasi-equilibria family (38). At that, $v_{\mathcal{e}}(x, t)=$ const. The operator $\mathcal{V}^{\prime}(\widetilde{f})$ acts as multiplying by a constant that is equal to $\left\langle\widetilde{u} P_{1}\right\rangle$, and the function $P_{1}$ arises from solving the problems (53). The system (49)-(51) gets the invariance with respect to the translations in the space and time. The spectral stability problem for the eigenmodes (43) depends on two functionals, the values of which it enters as parameters. These are the velocity of residual drift, $v_{e}=v_{e}(\widetilde{f})$, and one more functional that we define as follows.

$$
\mu: \tilde{f} \mapsto 1+\left\langle\widetilde{u} P_{1}\right\rangle .
$$

For these functionals, there are the explicit expressions specified by Formulae (37) (where $\Gamma_{+}=\Gamma_{+}(z)$ ) and (A20). At that, $\mu(0)=1, \mu(\widetilde{f})>0 \forall f=\widetilde{f}+\bar{f}$, and $v_{e}(0)=0$. All other parameters of the stability problem are independent of the external signal. Furthermore, let a be the effective amplitude that we have been defining in the line (26). Then $\mu \rightarrow+0$ and $v_{e}-c \rightarrow 0$ when $a \rightarrow \infty$, and the convergence to the limit values is exponential provided that the function (24) has no degenerate critical points on the $\eta$-axis.

Proof. The lemma follows from Lemma 1 and from the explicit expressions listed in Example 1 except for the assertion on the limit equalities. They arise from Laplace's asymptotics of the mentioned explicit expressions for $a \rightarrow \infty$. We will be discussing it in Appendix B.

Remark 2. The exponential convergence stated by Lemma 2 relies on a non-degeneracy condition imposed on the signal profile - that is, on the function denoted as $g$ that determines the shape of the signal-making wave following the definition (39). This condition holds for a generic profile, e.g., $g=\sin \eta$. 
Remark 3. Under hypothesis of Lemma 2, the analyticity of the drift operator follows from the explicit expressions for the drift velocity that we have been deriving while considering Example 1.

Let the signal obey the assumption of the Lemma 2. For a specific homogeneous quasi-equilibrium with densities $p_{e}$ and $q_{e}=1-p_{e}$ consider the spectral stability problem for the eigenmodes (43). This problem depends on the values of $v_{e}=v_{e}(\widetilde{f})$ and $\mu=\mu(\widetilde{f})$ and also on $\alpha, v, \delta, p_{e}$, which are independent of $f$. Define $\beta=\alpha^{2}$,

$$
c_{*}\left(p_{e}, \beta, v, \delta\right)=\sqrt{v\left(q_{e}+\delta \beta\right) \beta^{-1}}, q_{e}=1-p_{e} .
$$

Theorem 2. Under the assumption of the Lemma 2, consider a spectral stability problem for the eigenmodes (43) linked to a specific quasi-equilibrium with predators' density $p_{e}$. Then the following assertions hold.

(i) The corresponding neutral submanifold in the parametric space is bifoliated. The leaves of it are the graphs of the functions $\kappa=\mu^{-1}(\widetilde{f}) \kappa_{c}^{ \pm}\left(v_{e}, p_{e}, \beta, v, \delta\right)$ where $\beta=\alpha^{2}, v_{e}=v_{e}(\widetilde{f})$ and

$$
\kappa_{c}^{( \pm)}=\frac{c_{*}\left(\nu+q_{e}+\delta \beta\right)\left(c_{*} \pm\left|v_{e}\right|\right)}{p_{e} q_{e}} .
$$

On the neutral submanifold, the instability is oscillatory. Every eigenmode having the wavenumbers $\alpha= \pm \sqrt{\beta}$ is stable provided that the following inequality holds.

$$
\kappa<K=K\left(\widetilde{f}, p_{e}, \beta, v, \delta\right)=\mu^{-1}(\widetilde{f}) \kappa_{c}^{-}\left(v_{e}(\widetilde{f}), p_{e}, \beta, v, \delta\right),
$$

and there is an unstable eigenmode having the same wavenumbers and linked to the same quasi-equilibrium provided that $\kappa>K$.

(ii) Let $c>c_{*}\left(p_{e}, \beta, v, \delta\right)$. Then $\kappa_{c}^{-}\left(v_{e}, p_{e}, \beta, v, \delta\right)<0$ for every sufficiently large value of $a$ and the inequality (57) necessarily fails to hold; thus, the considered eigenmode is unstable for every admissible set of the other problem parameters; moreover, every eigenmode is unstable provided that $v_{e}^{2}-v \delta>0$ and the wavenumber of it obeys the inequality $\alpha^{2}>\beta_{*}=q_{e} v\left(v_{e}^{2}-v \delta\right)^{-1}$.

(iii) Let $c<c_{*}\left(p_{e}, \beta, v, \delta\right)$. Then there exits the threshold amplitude $a_{0}=a_{0}\left(p_{e}, \beta, v, \delta\right)$ such that the inequality (57) holds for every value of $a>a_{0}$. If, in addition, $c<\min _{\beta} c_{*}=\sqrt{v \delta}$ then there exits the threshold amplitudes $a_{1}=a_{1}(g, c, v, \delta)$ (where $g$ is the signal profile) such that

$$
\mathcal{K}_{*}^{-}(a, c, v, \delta)=\inf \left\{\mathcal{K}_{c}^{-}\left(v_{e}, p_{e}, \beta, v, \delta\right), 0<p_{e}<1, \beta>0\right\}>0, \quad \forall a>a_{1} .
$$

Let $a>a_{1}$. Define $K_{*}(a, c, v, \delta)=\mu^{-1}(\widetilde{f}) \kappa_{*}^{-}(a, c, v, \delta)$ Once $\kappa<K_{*}(a, c, v, \delta)$ the quasi-equilibria (38) get the collective stability in the sense that every such quasi-equilibrium is stable irrespective of the specific values of $p_{e}$ and $\beta$, provided that they obey the restrictions (44).

Proof. The assertions (ii) and (iii) are the corollaries to Lemma 2 and the equality (56). We place the proof of the latter in the second subsection of Appendix C.

Remark 4. The inequality $c>c_{*}$ and Lemma 2 entail the inequality $v_{e}^{2}>v \delta$ for every sufficiently high value of $a$. Indeed, $v_{e} \rightarrow c$ for $a \rightarrow \infty$ while $c>c_{*} \geq \min _{\beta} c_{*}=\sqrt{v \delta}$. Thus, $\beta_{*}>0$ under the conditions of the clause (ii).

Remark 5. By definitions (47) and (56), $\kappa_{c}^{+}>\kappa_{c}^{(0)}>\kappa_{c}^{-}$provided $v_{e}(\widetilde{f}) \neq 0$, and $\kappa_{c}^{+}=\kappa_{c}^{(0)}=\kappa_{c}^{-}$otherwise. Thus we can distinguish the upper and lower neutral leaves. By Theorem 2, the unstable eigenmodes already have arisen somewhere at the lower leaf when a point of the parametric space is approaching the upper leaf. Crossing the upper leaf never changes the stability of the corresponding quasi-equilibrium but the number of the associated unstable eigenmodes (see Appendix C.2 in Appendix C). Thus the upper leaf is not relevant to 
the stabilization and destabilization of quasi-equilibria. Nevertheless, it deserves a few words more, and we will continue discussing it in Section 6.

\section{Effect of Inhomogeneity: Stabilizing vs. Destabilizing}

In this section, we freeze the profile of the signal-making wave (39) and discuss the effects exerted from varying the speed and amplitude of it.

Let us assume for a while that the signal is stationary—that is, $c=0$, and

$$
f=\widetilde{f}=A g, g=g(\xi) .
$$

As we have been mentioning while discussing Example 2, such a signal does not produce a residual drift-that is, $v_{e}=0$. By Theorem 2, all we have been saying in Section 4.2 about the stability of homogeneous equilibria is also true regarding the quasi-equilibria upon replacing the critical value of the tactic efficiency, $\kappa_{c}$, by $\mu^{-1}(\widetilde{f}) \kappa_{c}^{(0)}$, where $\kappa_{c}^{(0)}$ is the quantity defined in (47). In particular, the quasi-equilibria get the collective stability provided that

$$
\kappa<\mu^{-1}(\widetilde{f}) \kappa_{*}^{(0)}(\nu, \delta) .
$$

It turns out that increasing the signal amplitude can make this inequality true. Indeed, for the stationary signals defined by the expression (59),

$$
\mu(\widetilde{f})=\frac{1}{\left\langle\mathrm{e}^{-a s}\right\rangle\left\langle\mathrm{e}^{a s}\right\rangle}, a=\frac{A}{v_{1} v_{2}}>0, s=-\partial_{\tilde{\zeta}}^{-2} \widetilde{f} .
$$

One can derive this formula from Lemma 1 by employing the calculations performed upon considering Example 2. Alternatively, one can employ the Formula (35). Then differentiating it with variable $z$ and setting $z=c=0$ again gives the expression (61). The expression (61) and inequality (60) together bring us at an explicit formulation of the criterion for the collective stability of quasi-equilibria that reads as

$$
\kappa<\left\langle\mathrm{e}^{-a s}\right\rangle\left\langle\mathrm{e}^{a s}\right\rangle \kappa_{*}^{0} .
$$

Since $\left\langle\mathrm{e}^{-a s}\right\rangle\left\langle\mathrm{e}^{a s}\right\rangle>1$ for every stationary signal (59), the criterion (62) implies that such a signal always enhances the collective stability compared to the case of homogeneity. The following example shows how powerful this stabilization can be.

Example 4. Let $f=A \sin \xi, A=$ const $>0$ and $c=0$. Then $s=\sin \xi$, and $\mu(\widetilde{f})=\left(\left\langle\mathrm{e}^{-a s}\right\rangle\left\langle\mathrm{e}^{a s}\right\rangle\right)^{-1}=$ $I_{0}^{-2}(a)<1$ where $I_{0}$ is the modified Bessel function of first kind. Consequently, the criterion (62) for the collective stability takes the following form: $\kappa<I_{0}^{2}(a) \kappa_{*}^{(0)}$. In this inequality the right-hand side grows exponentially when the effective amplitude, a, increases. Hence, increasing the signal level enhances the collective stability at an exponential rate.

Example 4 reveals exponential suppressing the tactic efficiency due to amplifying an external signal since the quantity $I_{0}^{-2}(a) \kappa$ plays the role of the tactic efficiency. Similarly, one can put the general criterion for the collective stability given by Theorem 2 as $\kappa \mu(\widetilde{f})<\kappa_{*}^{-}$. Here, the product $\kappa \mu(\widetilde{f})$ stands as the modified tactic efficiency. At that, $\mu(\widetilde{f})$ decays exponentially for $a \rightarrow+\infty$ by Lemma 2; thus, exponentially suppressing the tactic efficiency is typical for the stationary signals (59) and even for the traveling waves (39). It is not unexpected, except for the exponential acuity of the effect. Indeed, an intensive external signal is capable of distracting the predators from pursuing the prey. In particular, amplifying the generic stationary signal entails an exponential collective stabilization similar to that demonstrated by Example 4. However, it does not work so straightforwardly for the traveling waves. The matter of fact is that they produce drifting that modifies the value of $\kappa_{*}^{-}$, while the stationary signals cannot do so. 
From Theorem 2, it follows that amplifying the signal-making wave exerts the opposite effects depending on the relation of the wave speed, $c$, to an independent threshold denoted as $c_{*}$. Namely, for $c>c_{*}$, amplifying the signal leads to the total destabilization of the short-wavelength modes as described by clause (ii) of the theorem. Compared to the case of homogeneity, this is an utterly destabilizing effect, which sharply contrasts with the case of the stationary signal. For $c<c_{*}$, amplifying the signal stabilizes the quasi-equilibria in the same way as in the case of the stationary signal in accordance with clause (iii) of Theorem 2. Figures 1 and 2 illustrate the described changes.

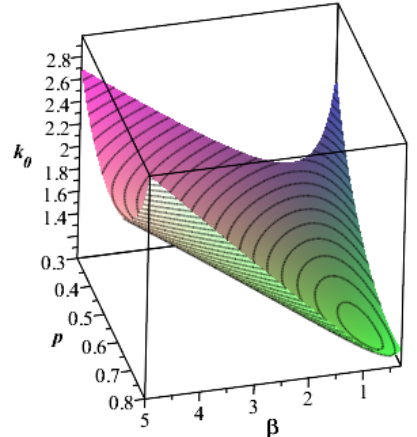

(a)

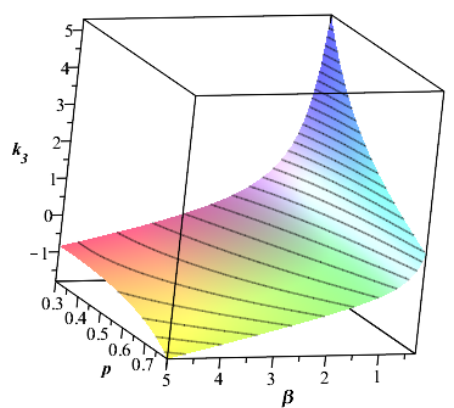

(d)

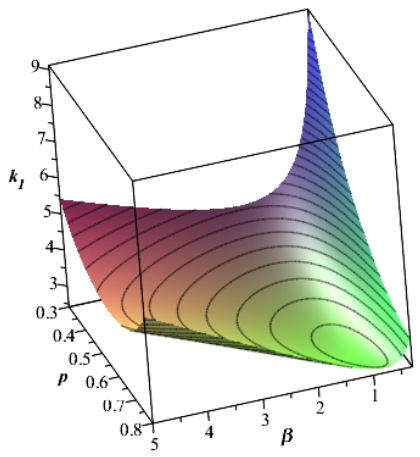

(b)

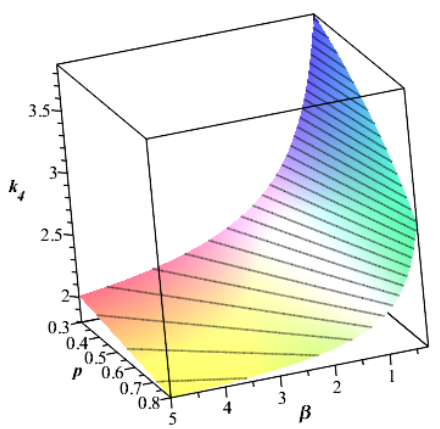

(e)

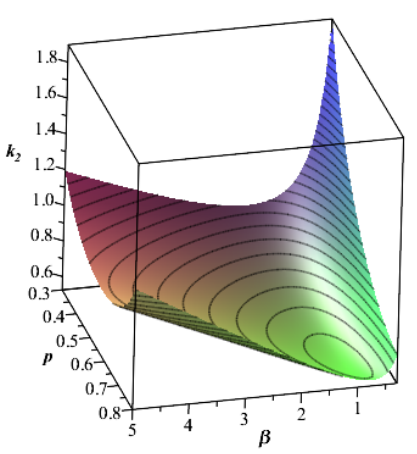

(c)

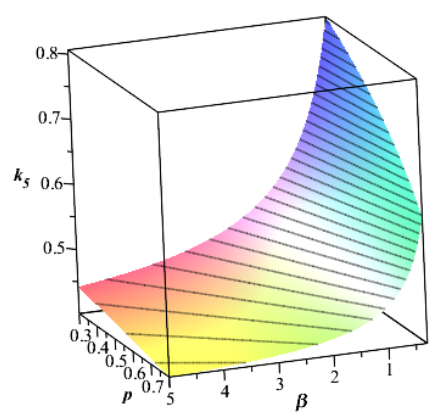

(f)

Figure 1. The boxes $(\mathbf{a}-\mathbf{d})$ show the areas of stability and instability in the space of parameters $p_{e}, \beta, \kappa$ for $\bar{\delta}=(1,1,0,0)$ by visualizing the neutral surface $\kappa=k_{j}, j=0,1,2,3$, where $k_{j}=$ $K\left(\tilde{f}_{j}, p_{e}, \beta, 1,1\right) / \kappa_{*}^{(0)}(1,1), j=0,1,2, k_{3}=\max \left(0, K\left(\widetilde{f}_{3}, p_{e}, \beta, 1,1\right)\right), \widetilde{f}_{m}=a_{m} \sin \left(\xi-c_{m} \tau\right), m=0,1,2,3$, $a_{0}=c_{0}=0, a_{1}=3, a_{2}=2, a_{3}=3.5, c_{1}=0.8, c_{2}=c_{3}=1.2$. Note that $\min _{\beta} c_{*}\left(p_{e}, \beta, 1,1\right)=1$. Hence, $c_{1}<c_{*}$ for every $p_{e}$ and $\beta$ while $c_{2}>c_{*}$ for some $p_{e}$ and $\beta$. Additionally, for every box in this figure, $v_{1}=v_{2}=1$ and $a=A$. Thus, the box (a) corresponds to the case of homogeneity and shows in fact $\kappa_{c}^{(0)}\left(p_{e}, \beta, 1,1\right) / \kappa_{*}^{(0)}(1,1)$. At that, $\kappa_{*}^{(0)}(1,1)=\kappa_{*}(1,1,0,0)=27 / 2$ and this is the minimum value of $\kappa_{c}^{(0)}=\left.\kappa_{c}\right|_{\bar{\delta}=(1,1,0,0)}$ attained at $p_{e}=\beta=2 / 3$. The boxes $(\mathbf{e}, \mathbf{f})$ in the lower row show the graphs of the functions $k_{4,5}=K\left(\tilde{f}_{1,2}, p_{e}, \beta, 1,1\right) / \kappa_{c}^{(0)}\left(p_{e}, \beta, 1,1\right)$.

Figure 1 illustrates the behavior of neutral surfaces $\kappa=K\left(\widetilde{f}, p_{e}, \beta, v, \delta\right)$ in $p_{e}, \beta, \kappa$-space for the frozen values of $v=\delta=1$. At that, the parameters $c$ and $a$ control the signal $\widetilde{f}$ and the values of them are varying. Since $\min _{\beta} c_{*}\left(p_{e}, \beta, v, \delta\right)=\sqrt{v \delta}=1$, the inequality $0<c<1$ entails the inequalities $c<c_{*} \forall \beta, p_{e}$, and the inequality $c>1$ entails the inequalities $c>c_{*}$ for some $\beta, p_{e}$. The boxes (a) to (d) in Figure 1 show the neutral surfaces for $v=\delta=1$ and several values of $c$ and $a$. The box (a) corresponds to $a=0$ and shows in fact $\kappa_{c}^{(0)}$. We have been discussing this picture in Section 4.2. Now it stands as an etalon. The other pictures show the changes due to increasing the parameter $a$ for $c>1$ and $c<1$ (we have placed the concrete values in the figure caption). At that, the boxes (e) and (f) reflect 
the relative changes by imaging the value of $K / \kappa_{c}^{(0)}$. Thus the boxes (b) and (e) depict the changes made by increasing the value of $a$ for $c<1$. We see that the shape of the neutral surface remains qualitatively the same while the threshold values of the tactic efficiency increase about two times at least for every value of the parameters $p_{e}$ and $\beta$ in the considered range. The boxes (c) and (f) show the changes made by a moderate increasing the value of $a$ for $c>1$. The shape of the neutral surface remains qualitatively the same in the considered range of $p_{e}$ and $\beta$, though the minimum is visibly less pronounced. At that, the threshold values of the tactic efficiency reduce themselves substantially everywhere and more than twice in some places. Further increasing the value of $a$ for the same $c$ causes dramatic changes in the shape of the neutral surface, as the box (d) tells us. The main difference is that the quantity $K$ is no more definite in sign and takes negative values for every sufficiently large value of the parameter $\beta$. Such a behavior corresponds to the total destabilizing of the shortwave eigenmodes.

Figure 2 illustrates the inverting of effect of the corresponding signal on the stability of quasi-equilibria due to varying the values of $c$ and $a$ for $v=\delta=1$. In Figure 2, the frame (a) shows what happens to a specific eigenmode for $p_{e}=\beta=2 / 3$. These values deliver the minimum value $\kappa_{*}^{(0)}=\kappa_{*}^{(0)}(1,1)=27 / 2$ to the threshold $\kappa_{c}^{(0)}=\kappa_{c}^{(0)}\left(p_{e}, \beta, 1,1\right)$. The partition of $c$, a-plane depicted there arises from the inequality $K(\widetilde{f}, 2 / 3,2 / 3,1,1)>\kappa_{*}^{(0)}(1,1)$. Thus, stabilizing (destabilizing) the considered mode compared to the case of homogeneity takes place in the area where this inequality is true (false). The former (the latter) is in burgundy red (navy blue) color. The red curve indicates the interface of these areas and the black curve corresponds to $c=c_{*}(2 / 3,2 / 3,1,1)=\sqrt{3 / 2}$. Note that the red curve approaches the black one upon increasing the value of $a$ in consistence with the limit behavior of $v_{e}$ asserted by Lemma 2 .

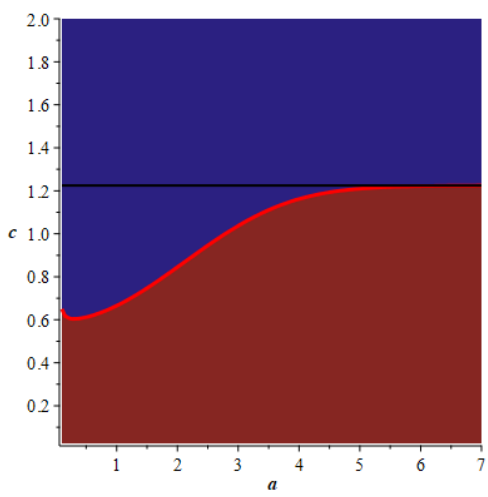

(a)

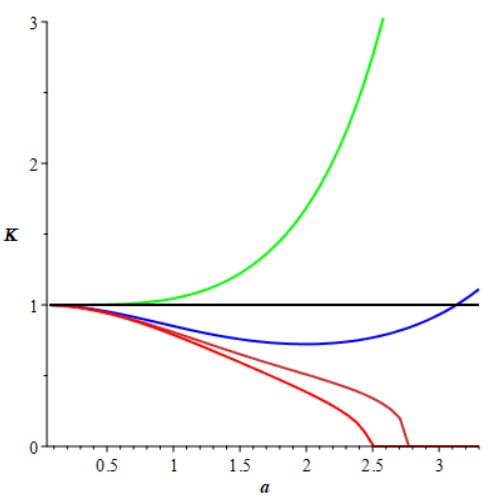

(b)

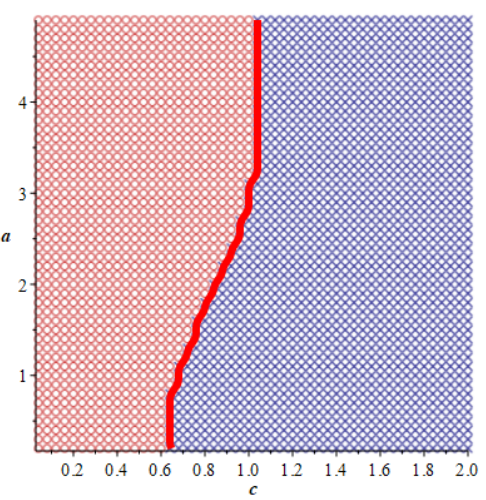

(c)

Figure 2. The figure depicts how the effect of the external signal turns upside down depending on the speed at which the signal-making wave propagates, $c$, and the amplitude of this wave, $a$. For all frames in this figure, $p_{e}=\beta=2 / 3, v_{1}=v_{2}=1$, and $a=A$. The frame (a) illustrates the partition of $c$, $a$-plane arises from the inequality $K(\widetilde{f}, 2 / 3,2 / 3,1,1)>\kappa_{*}^{(0)}(1,1)$, where $\widetilde{f}=a \sin (\xi-c \tau), \kappa_{*}^{(0)}(1,1)=27 / 2$ is the minimum value of $\kappa_{c}^{(0)}\left(p_{e}, \beta, 1,1\right)$ attained at $p_{e}=\beta=2 / 3$. The area where this inequality is true (false) is in burgundy red (navy blue ) color. The curve colored in red indicates the interface of these areas and the black one corresponds to $c=c_{*}(2 / 3,2 / 3,1,1)=\sqrt{3 / 2}$. The frame (c) shows the partition of $c$, $a$-plane by the inequality $K_{*}(\tilde{f}, 1,1)>\kappa_{*}^{(0)}(1,1)$. The area where this inequality holds (fails) is filled with the orange circles (navy blue diagonal crosses). The red curve indicates the interface of these areas. The frame $(\mathbf{b})$ shows the values of $\max \left(K_{*}\left(f_{j}, 2 / 3,2 / 3,1,1\right) / \kappa_{*}^{(0)}(1,1), 0\right)$ vs. $a$, where $f_{j}=a \sin \left(\xi-c_{j} \tau\right), j=1,2,3,4, c_{1}=3 / 5, c_{2}=1, c_{3}=1.1 \sqrt{3 / 2}, c_{4}=3 / 2$. The colors of the corresponding curves are green, blue, orange and red. The black curve corresponds to $f=0$. Note that $c_{*}(2 / 3,2 / 3,1,1)=\sqrt{3 / 2}$ and $\min _{\beta} c_{*}\left(\beta, p_{e}, 1,1\right)=1$.

The frames (b) and (c) in Figure 2 address the collective stability with the use of the quantities $K_{*}(a, c, v, \delta)$ and $\kappa_{*}^{(0)}(v, \delta)$, which give the threshold tactic efficiencies for losing the collective stability for $a \neq 0$ and $a=0$, correspondingly. The frame (c) shows the partition of $c$, $a$-plane by the inequality 
$K_{*}(a, c, 1,1)>\kappa_{*}^{(0)}(1,1)$. Enhancing (reducing) the collective stability compared to the case of homogeneity takes place in the area where this inequality holds (fails). The former (the latter) is filled with the orange circles (navy blue diagonal crosses). The red curve indicates the interface of these areas. Note that the interface curve gets to the line $c=1=\min _{\beta} c_{*}\left(p_{e}, \beta, 1,1\right)$ very closely upon increasing the value of $a$ (cf. clause (iii) in Theorem 2).

The frame (b) in Figure 2 shows the values of $\max \left(K_{*}(a, c, 1,1) / \kappa_{*}^{(0)}(1,1), 0\right)$ vs. $a$ for several values of $c$. The green, blue, orange and red colors correspond to an increasing tetrad of the values of $c$ in which the first (last) two values are smaller (greater) than $c_{*}$ (we have placed the concrete values in the figure caption). The black curve corresponds to $f=0$ and $K_{*}=\kappa_{*}^{(0)}$. Looking at the blue and green curves, we see that increasing the value $a$ for $c<c_{*}$ makes the threshold of the collective stability greater than in the case of homogeneity perhaps after some reducing. In contrast, two other curves show that increasing the value of $a$ for $c>c_{*}$ decreases the threshold of the collective stability up to the zero. At that, attaining the zero means the total destabilization. Anyway, the changes accelerate themselves very sharply for the greater values of $a$. This feature reminds us of the exponential asymptote of the quantities $\mu$ and $v_{e}$ for $a \rightarrow \infty$.

\section{Concluding Remarks}

The short-wavelength stabilization and destabilization of the quasi-equilibria resemble the effects of high-frequency vibrations widely known in the classical mechanics of particles or the continuous media. The examples are the upside-down pendulum and the counterparts of it emergent from the dynamics of the stratified fluid. The vibrations cause small-scale fluctuations of external force fields, e.g., the gravity. These fluctuations, on average, produce so-called added potential energy, which stands as the conductor of the effect of the vibrations on the large-scale smooth motions. The articles $[38,39]$ provide more details on this issue. The examples we have delivered above show that the small-scale environmental fluctuations exert the effect on the large-scale pattern formation by PKS systems in quite a different way, namely, by producing the drift of species. In particular, the two-leaf foliation of the neutral submanifold is a characteristic consequence of the drift because of breaking the mirror symmetry by it. When the drift arises, the double neutral eigenmode that is due to the mentioned symmetry splits itself into two simple ones which correspond to the lower and upper leaves. As we said in Remark 5, crossing the upper leaf cannot change the stability of the quasi-equilibrium but does change the number of the associated unstable eigenmodes. The bifurcations accompanying this changing can create various regimes, which can suddenly reveal themselves upon the evolution of finite perturbations for $\kappa_{c}^{+} \mu^{-1}>\kappa>\kappa_{c}^{-} \mu^{-1}$ (and for $\kappa>\kappa_{c}^{-} \mu^{-1}$, of course). These phenomena may become tractable in a two-parametric setting when the parameters, say, $c$ and $\mathcal{\kappa}-\left.K_{*}\right|_{c=0}$, are close to zero.

The large-scale drifting of a substance due to the small-scale fluctuations of the velocity transporting it is known as the Stokes drift. The effect of it on the long-term transport or mixing is the subject of continuing research; see, e.g., the article [40] for the details and references. In contrast, the effects exerted from Stokes' drift on the stability similar to those reported by the present research are not widely known.

Author Contributions: Conceptualization, A.M. and K.I.; formal analysis, A.M. and K.I.; investigation, A.M. and K.I.; methodology, A.M.; writing - original draft, A.M. All authors have read and agreed to the published version of the manuscript.

Funding: This research received no external funding.

Acknowledgments: Andrey Morgulis acknowledges the support from Southern Federal University (SFedU). Both authors are grateful to Inna Senina for the fruitful remarks.

Conflicts of Interest: The authors declare no conflict of interest. 


\section{Appendix A. Derivation of the Asymptotic Approximation}

This appendix aims at proving Theorem 1.

Proof of Theorem 1. We look for an asymptotic expansion of the solution to system (4)-(6) that reads as

$$
(u, p, q)=\sum_{k \geq 0} \omega^{-k}\left(u_{k}, p_{k}, q_{k}\right)(x, t, \xi, \tau), \quad \xi=\omega x, \tau=\omega t, \omega \rightarrow \infty .
$$

We require all the coefficients of series (A1) to be smooth functions in the coordinates $\xi, \tau$ on $\mathbb{T}^{2}$.

Assuming that the unknowns are the functions in the variables $x, t, \xi, \tau$, the system (4)-(6) takes the following form:

$$
\begin{gathered}
\omega\left(\left(\partial_{t}+\omega \partial_{\tau}\right) u-\left(\partial_{x}+\omega \partial_{\xi}\right)(\kappa q+f)+v u\right)=v_{1}\left(\partial_{x}+\omega \partial_{\xi}\right)^{2} u ; \\
\omega\left(\left(\partial_{t}+\omega \partial_{\tau}\right) p+\left(\partial_{x}+\omega \partial_{\xi}\right)(u p)\right)=v_{2}\left(\partial_{x}+\omega \partial_{\xi}\right)^{2} p ; \\
\left(\partial_{t}+\omega \partial_{\tau}\right) q-q(1-p-q)=\delta\left(\partial_{x}+\omega \partial_{\xi}\right)^{2} q .
\end{gathered}
$$

Replacing the unknowns in the system (A2)-(A4) by the series (A1) and collecting the terms of equal order in $\omega$ yields a sequence of equations. We will be solving them in sequence.

Collecting the terms of order $\omega^{2}$ in Equations (A2)-(A4) leads to the equations

$$
\begin{gathered}
q_{0 \xi \xi}=0 ; \\
u_{0 \tau}-v_{1} u_{0 \xi \xi}=f_{\xi} ; \\
p_{0 \tau}+\left(u_{0} p_{0}-v_{2} p_{0 \xi}\right) \xi=0 .
\end{gathered}
$$

There are no periodic solutions to Equation (A5) except for those independent of $\xi$. Hence

$$
q_{0}=q_{0}(x, t, \tau) .
$$

We have to determine the function (A8) at the subsequent steps.

We define the function $\widetilde{u}$ as the solution of Equation (A6) on $\mathbb{T}^{2}$ that vanishes on average in the sense of definition (7). Such a solution is unique. Since Equation (A6) is exactly the first equation of the system (13), we set $u_{0}=\bar{u}+\widetilde{u}$, where $\bar{u}=\left\langle u_{0}\right\rangle$ remains undefined and enters the homogenized system (15)-(17) as one of the unknowns. Thus, we have justified the leading term in the asymptotic approximation for $u$ given by (11), (13).

At the moment, we need the following

Lemma A1. Let $w=w(\xi, \tau)$ be a smooth function on $\mathbb{T}^{2}$. Consider the following problem

$$
\partial Q_{\tau}+\partial\left(w Q-\epsilon Q_{\xi}\right)_{\xi}=0, \quad\langle Q\rangle=1, \quad \epsilon=\text { const }>0,
$$

Then there exists a unique solution to the problem (A9) on $\mathbb{T}^{2}$.

We will prove this assertion at the end of this appendix.

We continue constructing the asymptotic expansion. By the above lemma, problem (14) has a unique solution $P=P(x, t, \xi, \tau)$. Hence, every solution to Equation (A7) reads as

$$
p_{0}=\bar{p}(x, t) P(x, t, \xi, \tau) .
$$

Thus we have got the leading term of asymptotic approximation (12) for the unknown $p$.

Now let us consider the terms of order $\omega$. It follows from Equations (A4) and (A8) that

$$
\delta q_{1 \xi \xi}=q_{0}
$$


Since the function $q_{0}$ does not depend on the variable $\xi$, the Equation (A11) has a periodic solution if and only if the function $q_{0}$ does not depend on $\tau$. Hence $q_{0}=\bar{q}(x, t)$ and we get the leading term of asymptotic approximation (10) for the unknown $q$. Further, every solution to Equation (A11) reads as

$$
q_{1}=q_{1}(x, t, \tau)
$$

Functions $q_{1}$ and $\bar{q}$ are still undefined. Note that the existence of a periodic solution to Equation (A11) justifies the error estimate (i.e., O-term) of the asymptotic approximation (10).

Given the equality (A12), collecting the terms of order $\omega$ in Equations (A2) and (A3) leads to the following equations

$$
\begin{gathered}
u_{1 \tau}-v_{1} u_{1 \xi \xi}=2 v_{1} u_{0 x \xi}+\left(\kappa q_{0}+\sigma f\right)_{x}-v u_{0}-u_{0 t} ; \\
p_{1 \tau}+\left(u_{0} p_{1}-v_{2} p_{1 \xi}\right)_{\xi}=2 v_{2} p_{0 x \xi}-p_{0 t}-\left(u_{0} p_{0}\right)_{x}-\left(u_{1} p_{0}\right)_{\xi}, \quad(\xi, \tau) \in \mathbb{T}^{2} .
\end{gathered}
$$

Averaging Equations (A13) and (A14) while keeping in mind the equality (A10) brings us at Equations (15) and (16). Similarly, proceeding with Equation (A4), we get Equation (17), that completes the homogenized system consisting of Equations (15)-(17). Resolving the homogenized system entails existence of the periodic solutions to Equations (A13) and (A14) and, consequently, justifies O-terms of the asymptotic approximations (11) and (12).

Proof of Lemma A1. Let $\mathrm{H}$ be the space of the Fourier series in $\xi$, $\tau$ with square-summable coefficients and let the notation of $\mathcal{L}: \mathrm{H} \rightarrow \mathrm{H}$ stand for the operator associated with the left-hand side of PDE (A9). We have to prove that

$$
\operatorname{dim} \operatorname{Ker} \mathcal{L}=1,\langle\chi\rangle \neq 0 \forall \chi \in \operatorname{Ker} \mathcal{L} \backslash\{0\}
$$

Let $\mathcal{L}^{*}$ denote the operator adjoint to $\mathcal{L}$ and let $\mathcal{J}: \mathrm{H} \rightarrow \mathrm{H}$ be the action of inversion $(\xi, \tau) \mapsto$ $(-\xi,-\tau)$. Define

$$
\breve{\mathcal{L}}^{*}=\mathcal{J} \mathcal{L}^{*} \mathcal{J}
$$

Then

$$
\breve{\mathcal{L}}^{*}: \varphi \mapsto\left(\partial_{\tau}-\epsilon \partial_{\xi \xi}\right) \varphi+w \partial_{\xi} \varphi
$$

Notice that PDE

$$
\left(\partial_{\tau}-\epsilon \partial_{\xi \xi}\right) \varphi+w \partial_{\xi} \varphi=0
$$

obeys the strong maximum and minimum principles (see, e.g., [41,42]). Hence

$$
\operatorname{Ker} \breve{\mathcal{L}}^{*}=\{\varphi \equiv \text { const }\}=\operatorname{Ker} \mathcal{L}^{*}
$$

Applying the unilateral strong maximum/minimum principles to PDE

$$
\left(\partial_{\tau}-\epsilon \partial_{\xi \xi}\right) \varphi+w \partial_{\xi} \varphi=1
$$

shows that neither equation $\breve{\mathcal{L}}^{*} \breve{\psi}=1$ nor equation $\mathcal{L}^{*} \psi=1$ has a solution belonging to $\mathrm{H}$. Consequently, the resolvent $\left(\mathcal{L}^{*}-\lambda \mathcal{I}\right)^{-1}, \lambda \in \mathbb{C}$, has a simple pole at the origin. Since this resolvent is compact, the pair of operators $\mathcal{L}^{*}$ and $\mathcal{L}$ obeys Fredhölm's theorems. Hence $\operatorname{dim} \operatorname{Ker} \mathcal{L}=1$. Furthermore, conjecturing that $\langle\chi\rangle=0$ for some $\chi \in \operatorname{Ker} \mathcal{L} \backslash\{0\}$ would imply the existence of solution to equation $\mathcal{L}^{*} \psi=$ const $\neq 0$ but this contradicts to what we have proved above.

\section{Appendix B. Proofs of Lemmas 1 and 2}

This appendix aims at completing the proofs of Lemmas 1 and 2 concerned with the structure of the spectral stability problem. 
Proof of Lemma 1. While beginning the proof in Section 4.3, we have reduced it to the analyticity of the drift operator near the origin. Thus we proceed with this issue. In Section 3, we have defined the drift operator as follows $\mathcal{V}(f): \bar{u} \mapsto\langle\widetilde{u} P\rangle$. Let $\mathcal{L}: \mathrm{H} \rightarrow \mathrm{H}$ be the operator that we have been defining in the course of proving Lemma A1. Let $w$ be the coefficient of the differential expression which we have been linking to this operator. The kernel of $\mathcal{L}^{*}$ consists of the identically constant functions for every $w$. We put $w=\sigma+\widetilde{w}$, where $\sigma=$ const, and $\langle\widetilde{w}\rangle=0$. Let $\Pi^{*}(\sigma)$ be the spectral projector onto $\operatorname{ker} \mathcal{L}^{*}$. Then $\Pi^{*}(\sigma): g \mapsto\langle g Q\rangle$ where $Q$ is the solution to equation $\mathcal{L} Q=0$ satisfying the condition $\langle Q\rangle=1$. Hence, the well-known result in the perturbation theory for the linear operators implies that the operator-valued function $\Pi^{*}(\sigma)$ is analytic in some neighborhood of the origin. Then the mapping $\sigma \mapsto \Pi^{*}(\sigma) \widetilde{w}$ is analytic too. Hence, we arrive at the analyticity of the drift operator since $\langle\widetilde{u} P\rangle=\Pi^{*}(\bar{u}) \widetilde{u}$.

Proof of Lemma 2. We proceed with what we have begun in Section 4.3. We have been saying that the lemma follows from the Lemma 1 except for the assertions regarding the limits of the functionals $v_{e}$ and $\mu$ for $a \rightarrow \infty$. To prove them, let us derive Laplace's estimates for the exponential integrals denoted as $\Gamma_{ \pm}$as they enter the explicit expressions for the residual drift velocity obtained upon considering the Example 1. We have defined these integrals by Formula (30) using also the expressions (24) and (26)-(28). Since we have been restricting ourselves within the class of signals defined by equality (39), $\Gamma_{ \pm}=\Gamma_{ \pm}(z), \pm z>0$ where we have to put $z=c / v_{2}$ as we consider the residual drift.

Let $c>0$. Then we have to estimate integral $\Gamma_{+}$that enters the Formula (37) for the residual drift velocity. We put this integral into a slightly different form that is more convenient for applying the Laplace method, namely,

$$
\frac{1}{2 \pi} \int_{(0, \infty) \times(0,2 \pi)} \mathrm{e}^{-z \sigma} \mathrm{e}^{a S(\eta, \sigma)} d \eta d \sigma, \quad S(\eta, \sigma)=s(\eta)-s(\eta-\sigma), z>0 .
$$

Here we consider function $s$ as a given one, and we assume that it is $2 \pi$-periodic, vanishing on average and has no degenerated critical points. The periodicity allows us to reduce the domain of integration of integral (A16) to the following cylinder

$$
C=\{(\eta, \sigma) \in \mathbb{S} \times(0,2 \pi)\}
$$

and we arrive at estimating the following integral

$$
\int_{C} \mathrm{e}^{-z \sigma} \mathrm{e}^{a S(\eta, \sigma)} d \eta d \sigma
$$

The local maximizers of $S$ on the whole $(\eta, \sigma)$-plain are

$$
(\eta, \sigma) \in \mathbb{R}^{2}: s^{\prime}(\eta)=s^{\prime}(\eta-\sigma)=0, \quad s^{\prime \prime}(\eta) s^{\prime \prime}(\eta-\sigma)<0 .
$$

The last inequality means that no maximizers belong to $\partial C$. Then the leading term in Laplace's asymptotic approximation of integral (A17) for $a \rightarrow \infty$ reads as

$$
\frac{2 \pi \mathrm{e}^{a \operatorname{osc}(s)}}{a} \sum_{(x, y) \in M} \frac{\mathrm{e}^{-z(x-y)}}{\sqrt{-s^{\prime \prime}(x) s^{\prime \prime}(y)}}
$$

where

$$
\operatorname{osc}(s)=\sup _{\mathbb{R}} s-\inf _{\mathbb{R}} s, \quad M=\{(x, y): 0<x \leq 2 \pi, 0<x-y<2 \pi, \operatorname{osc}(s)=s(x)-s(y)\} .
$$


Coming back to the integral (A16) brings us at the following estimate.

$$
\Gamma_{+}(z) \sim \frac{\mathrm{e}^{a \operatorname{osc}(s)}}{a\left(1-\mathrm{e}^{-2 \pi z}\right)} \sum_{(x, y) \in M} \frac{\mathrm{e}^{-z(x-y)}}{\sqrt{-s^{\prime \prime}(x) s^{\prime \prime}(y)}}, \quad a \rightarrow \infty, \quad z=c / v_{2}>0 .
$$

This estimate and expression (37) entail exponential convergence $v_{e} \rightarrow c>0$ for $a \rightarrow \infty$. The exponential decay for the functional $\mu$ follows from the equality

$$
\mu(\widetilde{f})=\frac{1}{\Gamma_{+}^{2}\left(\frac{c}{v_{2}}\right)} \int_{0}^{\infty} R(\sigma) \mathrm{e}^{-\frac{c \sigma}{v_{2}}} \sigma d \sigma=\left.\frac{d \Gamma_{+}^{-1}(z)}{d z}\right|_{z=\frac{c}{v_{2}}}, \quad c>0,
$$

Note that these considerations cover the limit of $c \rightarrow+0$. Alternatively, one can tackle the case of $c=0$ separately by deriving Laplace's estimates for the mean values that enter the Formula (61).

The definition of $R$ given by equalities (26) and (27) entails the positiveness of the integrand in the integral (A20). Hence, $\mu(\widetilde{f})>0$.

Example A1. Let $s(\eta)=\sin \eta$. Then $\operatorname{osc}(s)=2, M=\{(\pi / 2,-\pi / 2)\}$, and estimate (A19) reads as

$$
\Gamma_{+}(z) \sim \frac{\mathrm{e}^{2 a} \mathrm{e}^{-\pi z}}{a\left(1-\mathrm{e}^{-2 \pi z}\right)}, \quad a \rightarrow \infty, \quad z=c / v_{2}>0 . .
$$

Differentiating this estimate gives the asymptotic for the value of $\mu(\widetilde{f})$. The limit of this asymptotic for $c \rightarrow+0$ is nothing else than the leading term in the asymptotic of the function $I_{0}^{-2}(a), a \rightarrow \infty$, in consistence with the Example 4.

\section{Appendix C. Linear Stability}

This appendix aims at the details of the linear stability analysis for the equilibria and quasi-equilibria.

\section{Appendix C.1. Equilibria}

Let us choose an equilibrium out of the family (38) by specifying the value of the family parameter denoted as $p_{e}$. The eigenvalues corresponding to eigenmode (43) having a specific wavenumber $\alpha$ are the solutions to the following algebraic equation.

$$
\begin{gathered}
\lambda^{3}+\left(D_{1}+D_{2}+D_{3}\right) \lambda^{2}+\left(D_{2} D_{3}+D_{1} D_{3}+D_{1} D_{2}\right) \lambda+D_{1} D_{2} D_{3}+\beta \kappa p_{e} q_{e}=0, \\
D_{1}=v+\beta \delta_{1} ; D_{2}=\beta \delta_{2} ; D_{3}=q_{e}+\beta \delta ; \beta=\alpha^{2}, p_{e}+q_{e}=1
\end{gathered}
$$

Given the restrictions (44), all the coefficients of the polynomial on the left-hand side of Equation (A21) are strictly positive. Consequently, the roots of this polynomial are neither positive nor zero. Hence, neither unstable nor neutral eigenmode corresponds to a real eigenvalue.

It follows from the Routh-Hurwitz theorem that the necessary and sufficient condition for belonging all the roots of a polynomial (A21) to the open left half-plane of the complex plane reads as

$$
\left(D_{1}+D_{2}+D_{3}\right)\left(D_{2} D_{3}+D_{1} D_{3}+D_{1} D_{2}\right)>D_{1} D_{2} D_{3}+\beta \kappa p_{e} q_{e} .
$$

This inequality allows a more compact form, namely:

$$
\left(D_{1}+D_{2}\right)\left(D_{1}+D_{3}\right)\left(D_{2}+D_{3}\right)>\beta \kappa p_{e} q_{e} .
$$

Since Equation (A21) is of the third degree, replacing the last inequality by equality is necessary and sufficient for belonging the root of the polynomial (A21) to the imaginary axis, and this root 
cannot be zero. Given this observation, we find the instability at the magnitude of the tactic efficiency specified by Formulae (45).

\section{Appendix C.2. Quasi-Equilibria}

In this subsection, we prove Theorem 2.

Proof of Theorem 2. Acting the same way as in the case of equilibria, we arrive at the characteristic polynomial that reads as

$$
\lambda^{3}+\left(i U+v+q_{e}+\delta_{q} \beta\right) \lambda^{2}+\left(\left(i U+q_{e}+\delta_{q} \beta\right) v+i U\left(q_{e}+\delta_{q} \beta\right)\right) \lambda+i v U\left(q_{e}+\delta_{q} \beta\right)+\beta p_{e} q_{e} \mu(\widetilde{f}) \kappa .
$$

Here $\beta=\alpha^{2}, \alpha$ stands for the eigenmode wavenumber, $U=\alpha v_{e}, v_{e}$ stands for the drift velocity determined by equality (33). For the sake of convenience, we write this polynomial in more compact form, namely,

$$
\lambda^{3}+\left(i U+d_{1}\right) \lambda^{2}+\left(d_{2}+i U d_{1}\right) \lambda+i U d_{2}+r
$$

where

$$
d_{1}=v+q_{e}+\delta \beta, d_{2}=v\left(q_{e}+\delta \beta\right), r=\beta p_{e} q_{e} \mu(\widetilde{f}) \kappa .
$$

Note that $r>0, d_{1}>0$ and $d_{2}>0$ for every admissible set of the problem parameters (The admissibility of the problem parameters presumes the positiveness of the values of $\kappa$, and $\mu(\widetilde{f})>0$ ).

We employ the complex-valued version of Hurwitz's theorem to count the roots belonging to the right complex semi-plane, and it brings us at the chain of the Hankel's matrix minors that reads as

$$
1, d_{1}, d_{1}\left(d_{2} d_{1}-r\right), r\left(\left(r-d_{1} d_{2}\right)^{2}-U^{2} d_{1}^{2} d_{2}\right) .
$$

For this chain, there are four generic distributions of signs of the minors, namely

$$
++++, \quad+++-, \quad++--, \quad++-+ \text {. }
$$

Switching between the diagrams listed here corresponds to crossing certain submanifolds in the space of the problem parameters. These are the graphs of functions $\kappa=\mu^{-1}(\widetilde{f}) \kappa_{c}^{(j)}\left(v_{e}, p_{e}, \beta, v, \delta\right)$, $j \in\{+,-, 0\}$, where the function $\kappa_{c}^{(0)}$ and $\kappa_{c}^{( \pm)}$are defined by equalities (47) and (56) correspondingly. Note that $\kappa_{c}^{-}<\kappa_{c}^{(0)}<\kappa_{c}^{+}$for every admissible set of the problem parameters provided that $v_{e} \neq 0$. If $v_{e}=0$ then $\kappa_{c}^{-}=\kappa_{c}^{(0)}=\kappa_{c}^{+}$. Therefore, when the effective tactic coefficient passes through the values of $\kappa_{c}^{-}, \kappa_{c}^{(0)}$, and $\kappa_{c}^{+}$in the ascending order, the transitions between the above diagrams go on from left to right, provided that the values of all other parameters stay unaltered. Hence, the number of roots in the right complex semi-plane takes the values of 0,1 and 2 provided that the value of $\bar{\kappa}$ belongs to intervals $\left(0, \kappa_{c}^{-}\right),\left(\kappa_{c}^{-}, \kappa_{c}^{+}\right)$and $\left(\kappa_{c}^{+}, \infty\right)$ correspondingly. Changing this number is due to changing the sign of the senior minor of Hankel's matrix. Therefore, crossing the imaginary axis by the root of the characteristic polynomial at some non-zero point happen each time the effective tactic coefficient passes through one of the values of $\kappa_{c}^{ \pm}$. Hence, the oscillatory instability occurs at this moment. No changing the number of unstable roots happens upon passing through the value of $\kappa_{c}^{(0)}$.

For $\kappa=\kappa_{c}^{ \pm}$and $v_{e}>0$ the neutral eigenmode is proportional to

$$
\mathrm{e}^{i \sqrt{\beta}\left(x \pm c_{*} t\right)},
$$

and every eigenmode appears together with the complex-conjugated one. For $v_{e}<0$, it suffices to substitute $x \pm c_{*} t$ by $x \mp c_{*} t$. 
Remark A1. The inequalities $\left|v_{e}\right|>c_{*}$ or $\left|v_{e}\right|<c_{*}$ are equivalent to $\kappa_{c}^{-}<0$ or $\kappa_{c}^{-}>0$. The negative values of $\kappa_{c}^{-}$for a specific wavelength exclude the diagram ++++ . Hence, the eigenmode with such a wavelength is unstable.

\section{References}

1. Ivanitskii, G.R.; Medvinskii, A.B.; Tsyganov, M.A. From the dynamics of population autowaves generated by living cells to neuroinformatics. Physics-Uspekhi 1994, 37, 961-989. [CrossRef]

2. Horstmann, D. From 1970 until present: The Keller-Segel model in chemotaxis and its consequences. Jahresber. Deutsch. Math.-Verein. 2004, 106, 51-69.

3. Hillen, T.; Painter, K.J. A user's guide to PDE models for chemotaxis. J. Math. Biol. 2015, 58, 183. [CrossRef] [PubMed]

4. Bellomo, N.; Bellouquid, A.; Tao, Y.; Winkler, M. Toward a mathematical theory of Keller-Segel models of pattern formation in biological tissues. Math. Models Methods Appl. Sci. 2015, 25, 1663-1763. [CrossRef]

5. Berezovskaya, F.S.; Karev, G.P. Bifurcations of travelling waves in population taxis models. Physics-Uspekhi 1999, 42, 917-929. [CrossRef]

6. Berezovskaya, F.S.; Karev, G.P. Parametric portraits of travelling waves of population models with polynomial growth and auto-taxis rates. Nonlinear Anal. Real World Appl. 2000, 1, 123-136. [CrossRef]

7. Tsyganov, M.A.; Brindley, J.; Holden, A.V.; Biktashev, V.N. Quasisoliton interaction of pursuitevasion waves in a predator-prey system. Phys. Rev. Lett. 2003, 91, 218102. [CrossRef]

8. Tsyganov, M.A.; Brindley, J.; Holden, A.V.; Biktashev, V.N. Soliton-like phenomena in one-dimensional cross-diffusion systems: A predator-prey pursuit and evasion example. Phys. D Nonlinear Phenom. 2004, 197, 18-33. [CrossRef]

9. Tsyganov, M.A.; Biktashev, V.N. Half-soliton interaction of population taxis waves in predator-prey systems with pursuit and evasion. Phys. Rev. E 2004, 70, 031901. [CrossRef]

10. Horstmann, D.; Stevens, A. A constructive approach to traveling waves in chemotaxis. J. Nonlinear Sci. 2004, 14, 1-25. [CrossRef]

11. Govorukhin, V.; Morgulis, A.; Tyutyunov, Y. Slow taxis in a predator-prey model. Dokl. Math. 2000, $61,420-422$.

12. Arditi, R.; Tyutyunov, Y.; Morgulis, A.; Govorukhin, V.; Senina, I. Directed movement of predators and the emergence of density-dependence in predator-prey models. Theor. Popul. Biol. 2001, 59, 207-221. [CrossRef] [PubMed]

13. Painter, K.J.; Hillen, T. Spatio-temporal chaos in a chemotaxis model. Phys. D Nonlinear Phenom. 2011, 240, 363-375. [CrossRef]

14. Pearce, I.G.; Chaplain, M.A.J.; Schofield, P.G.; Anderson, A.R.A.; Hubbard, S.F. Chemotaxis-induced spatio-temporal heterogeneity in multi-species host-parasitoid systems. J. Math. Biol. 2007, 55, 365-388. [CrossRef] [PubMed]

15. Wang, Q.; Yang, J.; Zhang, L. Time-periodic and stable patterns of a two-competing-species Keller-Segel chemotaxis model: Effect of cellular growth. Discret. Contin. Dyn. Syst. B 2017, 22, 3547-3574. [CrossRef]

16. Southall, B.L.; Finneran, J.J.; Reichmuth, C.; Nachtigall, P.E.; Ketten, D.R.; Bowles, A.E.; Ellison, W.T.; Nowacek, D.P.; Tyack, P.L. Marine Mammal Noise Exposure Criteria: Updated Scientific Recommendations for Residual Hearing Effects. Aquat. Mamm. 2019, 45, 125-232 [CrossRef]

17. Hsu, A.C.; Boustany, A.M.; Roberts, J.J.; Chang, J.; Halpin, P.N. Tuna and swordfish catch in the U.S. northwest Atlantic longline fishery in relation to mesoscale eddies. Fish. Oceanogr. 2015, 24, 508-520. [CrossRef]

18. Royer, F.; Fromentin, J.M.; Farrugio, H.; Gaspar, P. Determining bluefin tuna habitat through frontal features in the Mediterranean Sea. Collect. Vol. Sci. Pap. 2005, 58, 1275-1284.

19. Reese, D.C.; O'Malley, R.T.; Brodeur, R.D.; Churnside, J.H. Epipelagic fish distributions in relation to thermal fronts in a coastal upwelling system using high-resolution remote-sensing techniques. ICES J. Mar. Sci. 2011, 68, 1865-1874. [CrossRef]

20. Kratina, P.; Lecraw, R.M.; Ingram, T.; Anholt, B.R. Stability and persistence of food webs with omnivory: Is there a general pattern? Ecosphere 2012, 3, 1-18. [CrossRef]

21. Landau, L.D.; Lifshitz, E.M. Mechanics, 3rd ed.; Elsivier: Amsterdam, The Netherlands, 1982; 224p. 
22. Allaire, G. A brief introduction to homogenization and miscellaneous applications. In ESAIM: Proceedings; EDP Sciences: Les Ulis, France, 2012; Volume 37, pp. 1-49.

23. Allaire, G. Homogenization and two-scale convergence. Siam J. Math. Anal. 1992, 23, 1482-1518. [CrossRef]

24. Black, T. Boundedness in a Keller-Segel system with external signal production. J. Math. Anal. Appl. 2017, 446, 436-455. [CrossRef]

25. Issa, T.B.; Shen, W. Persistence, coexistence and extinction in two species chemotaxis models on bounded heterogeneous environments. J. Dyn. Differ. Equ. 2019, 31, 1839-1871. [CrossRef]

26. Yurk, B.P.; Cobbold, C.A. Homogenization techniques for population dynamics in strongly heterogeneous landscapes. J. Biol. Dyn. 2018, 12, 171-193. [CrossRef] [PubMed]

27. Tello, I.J.; Wrzosek, D. Predator-prey model with diffusion and indirect prey-taxis. Math. Models Methods Appl. Sci. 2016, 26, 2129-2162. [CrossRef]

28. Li, U.; Tao, Y. Boundedness in a chemotaxis system with indirect signal production and generalized logistic source. Appl. Math. Lett. 2018, 77, 108-113. [CrossRef]

29. Tyutyunov, Y.; Titova, L.; Senina, I. Prey-taxis destabilizes homogeneous stationary state in spatial Gause-Kolmogorov-type model for predator-prey system. Ecol. Complex. 2017, 31, 170-180. [CrossRef]

30. Dolak, Y.; Hillen, T. Cattaneo models for chemosensitive movement: Numerical solution and pattern formation. J. Math. Biol. 2003, 46, 461-478. [CrossRef]

31. Filbet, F.; Laurencot, P.; Perthame, B. Derivation of hyperbolic models for chemosensitive movement. J. Math. Biol. 2005, 50, 189-207. [CrossRef]

32. Iooss, G.; Joseph, D.D. Elementary Stability and Bifurcation Theory; Springer Science \& Business Media: Berlin, Germany, 2012; 285p.

33. Arnold, V.I.; Afrajmovich, V.S.; Il'yashenko, Y.S.; Shil'nikov, L.P. Dynamical Systems V: Bifurcation Theory and Catastrophe Theory; Springer Science \& Business Media: Berlin, Germany, 2013; Volume 5, 227p.

34. Haragus, M.; Iooss, G. Local Bifurcations, Center Manifolds, and Normal Forms in Infinite-Dimensional Dynamical Systems; Springer Science \& Business Media: Berlin, Germany, 2010; 329p.

35. Yudovich, V. Cycle-creating bifurcation from a family of equilibria of a dynamical system and its delay. J. Appl. Math. Mech. 1998, 62, 19-29. [CrossRef]

36. Li, C.; Wang, X.; Shao, Y. Steady states of a predator-prey model with prey-taxis. Nonlinear Anal. Theory Methods Appl. 2014, 97, 155-168. [CrossRef]

37. Berezovskaya, F.S.; Novozhilov, A.S.; Karev, G.P. Families of traveling impulses and fronts in some models with cross-diffusion. Nonlinear Anal. Real World Appl. 2008, 9, 1866-1881. [CrossRef]

38. Yudovich, V. The dynamics of vibrations in systems with constraints. Dokl. Phys. 1997, 42, 322-325.

39. Vladimirov, V. On vibrodynamics of pendulum and submerged solid. J. Math. Fluid Mech. 2005, 7, S397-S412. [CrossRef]

40. Vladimirov, V. Two-Timing Hypothesis, Distinguished Limits, Drifts, and Pseudo-Diffusion for Oscillating Flows. Stud. Appl. Math. 2017, 138, 269-293. [CrossRef]

41. Nirenberg, L. A strong maximum principle for parabolic equations. Comm. Pure Appl. Math. 1953, 6, 167-177. [CrossRef]

42. Landis, E.M. Second Order Equations of Elliptic and Parabolic Type; American Mathematical Soc.: Providence, RI, USA, 1997; 203p.

Publisher's Note: MDPI stays neutral with regard to jurisdictional claims in published maps and institutional affiliations.

(C) 2020 by the authors. Licensee MDPI, Basel, Switzerland. This article is an open access article distributed under the terms and conditions of the Creative Commons Attribution (CC BY) license (http:/ / creativecommons.org/licenses/by/4.0/). 